Synthesis and biological evaluation of some stilbene derivatives

Subhas Somalingappa Karki, Santosh Ramarao Bhutle, Subhas Sahoo, Ratnakar Reddy, Jan Balzarini, Erik De Clercq \& Satyanarayana Y. Darji

Medicinal Chemistry Research

ISSN 1054-2523

Volume 20

Number 8

Med Chem Res (2011) 20:1349-1356

DOI 10.1007/s00044-010-9484-1

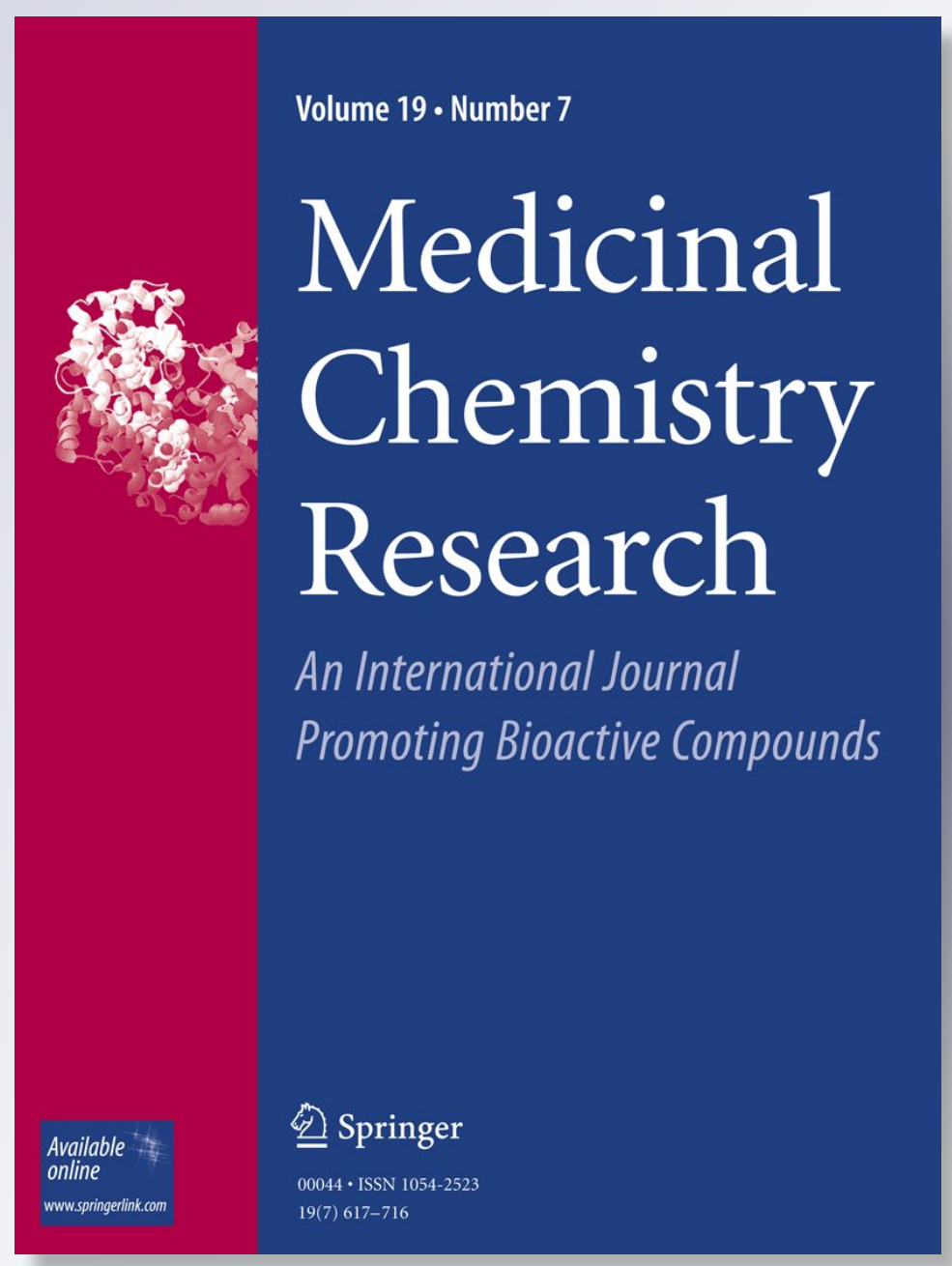

是 Springer 
Your article is protected by copyright and all rights are held exclusively by Springer Science+Business Media, LLC. This e-offprint is for personal use only and shall not be selfarchived in electronic repositories. If you wish to self-archive your work, please use the accepted author's version for posting to your own website or your institution's repository. You may further deposit the accepted author's version on a funder's repository at a funder's request, provided it is not made publicly available until 12 months after publication. 


\title{
Synthesis and biological evaluation of some stilbene derivatives
}

\author{
Subhas Somalingappa Karki • Santosh Ramarao Bhutle • \\ Subhas Sahoo $\cdot$ Ratnakar Reddy $\cdot$ Jan Balzarini • \\ Erik De Clercq $\cdot$ Satyanarayana Y. Darji
}

Received: 5 August 2010/Accepted: 19 October 2010/Published online: 5 November 2010

(C) Springer Science+Business Media, LLC 2010

\begin{abstract}
Several trans and cis stilbenes with substitution on the olefinic bridge were synthesized and characterized by IR, NMR and mass spectroscopy in an effort to obtain substances that could be more readily formulated. All the synthesized compounds were screened against Molt4/C8, CEM and L1210 cell lines. None of these compounds were endowed with pronounced cytostatic activity. However, Schiff derivatives emerged as cytostatic agents $\left(\mathrm{IC}_{50}\right.$ : $0.77-10 \mu \mathrm{g} / \mathrm{ml}$ ) that deserve further investigation.
\end{abstract}

Keywords Stilbenes $\cdot$ Cytostatic $\cdot$ Schiff $\cdot$ Olefine

\section{Introduction}

Cancer is one of the main reasons of death in both men and women. One promising natural product is resveratrol (3,4',5-trihydroxy-trans-stilbene) (RSV), a phytoalexin found in grapes and in red wine. The interest in this molecule has considerably increased in the last 10-12 years. The anticancer activity of resveratrol was first revealed by its ability to reduce the incidence of carcinogen-induced development of cancers in experimental animals (Jang et al., 1997; Dong, 2003). Since then, it has been demonstrated that it possesses chemopreventive and cytostatic

S. S. Karki $(\bowtie) \cdot$ S. R. Bhutle $\cdot$ S. Sahoo $\cdot$ R. Reddy

S. Y. Darji

Department of Pharmaceutical Chemistry, KLE University's

College of Pharmacy, Bangalore 560010, Karnataka, India

e-mail: subhasskarki@gmail.com

J. Balzarini · E. De Clercq

Rega Institute for Medical Research, Katholieke Universiteit Leuven, B-3000 Leuven, Belgium properties via the inhibition of tumor initiation, promotion and progression (Jang et al., 1997). It has also been demonstrated that RSV inhibits ribonucleotide reductase catalyzing the rate limiting step of de novo DNA synthesis (Fontecave, 1998). It exerts a nonselective cyclooxygenase1 (COX-1) and cyclooxygenase-2 (COX-2) inhibition (Jang and Pezzuto, 1999). The antioxidant properties of resveratrol have been related to its polyphenolic nature, especially to the presence of hydroxyl groups (Wright et al., 2001; Stivala et al., 2001). Phenolic groups are also known as an important structural determinant for estrogen receptor (ER) binding (Sadler et al., 1998; Ekena et al., 1998; Gao et al., 1999). Various trans stilbene compounds were reported to be inhibitors of Cytochrome P450 (CYP). Resveratrol showed an inhibitory effect on human CYP1A1 and CYP1B1 (Chun et al., 1999; Chang et al., 2000). Recently, rhapontigenin, a natural hydroxystilbene, showed a strong selectivity of CYP1A1 inhibition (Chun et al., 2001), and it was found that the selectivities and inhibitory potency of stilbene compounds tested against CYP1s were sensitive to the substitution patterns on the trans stilbene template. Cushman et al. (1992) have synthesized and evaluated analogues of (Z)-1-(4-methoxyphenyl)-2-(3,4,5trimethoxyphenyl)ethene as potential cytotoxic and antimitotic agents. Lion et al. (2005) synthesized a novel family of monohydroxylated $(E)$-stilbenes and studied their ability to inhibit the growth and induce apoptosis in human tumor cell lines. Heynekamp et al. (2006) synthesized various substituted trans stilbene including analogue of resveratrol and proved that they inhibit the human tumor necrosis factor $\alpha$-induced activation of transcription factor nuclear factor kappaB. Sangjun et al. (2009) synthesized cis stilbene derivative related to VIOXX and studied for their inhibitory effects on cell cycle progression and anti-estrogenicity in human adenoma breast cancer MCF-7 cells. 
Moran et al. (2009) synthesized fluorinated analogues of resveratrol and assayed on a variety of cell lines, primarily the non-small lung carcinoma cell line DLKP-A.

In this article we performed isosteric modifications of resveratrol by keeping the stilbene backbone of RSV to design substances that could be more readily formulated by (i) replacing the hydroxyl by various functional groups and (ii) introducing substituents on olefinic carbons and (iii) Schiff bases derivatives.

\section{Results and discussion}

\section{Chemistry}

Wittig reaction was carried out by using phosphonium chloride (2) with aryl aldehyde in benzene in the presence of sodium hydride. Excess of sodium hydride was quenched by addition of methanol and then chloroform and water were added. After evaporating, organic layer and residue were purified by preparative TLC using 5\% ethanol in hexane as the eluent (3a-f) (Scheme 1, Table 1).

Several derivatives containing acidic and basic functional groups were prepared in an attempt to make compounds that were more soluble in water and therefore be formulated more easily (Cushman et al., 1992). Base-catalyzed condition of phenyl acetic acid (4) with aryl aldehyde (5) in the presence of triethylamine gave the carboxylic acid (6a-c) (Scheme 2, Table 2). Reaction of $\mathrm{SOCl}_{2}$ with the carboxylic acid (6) in refluxing benzene gave the corresponding acid chlorides (7), which, upon subsequent reaction with appropriate amines, gave compounds (8a-g) (Scheme 2, Table 2). 9a and b derivatives were prepared by compound $\mathbf{6}$ with methanol using catalytic $\mathrm{H}_{2} \mathrm{SO}_{4}$.

Several Schiff derivatives (12a-d) were prepared as bioisosteric compounds of stilbene by refluxing aldehyde (10) and aromatic amines (11) in toluene in a Dean stark apparatus (Scheme 3, Table 3).

Scheme 1 General procedure for the preparation of 3a-f reagents and conditions: $e \mathrm{CH}_{3} \mathrm{CN}$, triphenyl phosphine, $f \mathrm{NaH}$, aryl aldehyde, benzene, $0-5^{\circ} \mathrm{C}$

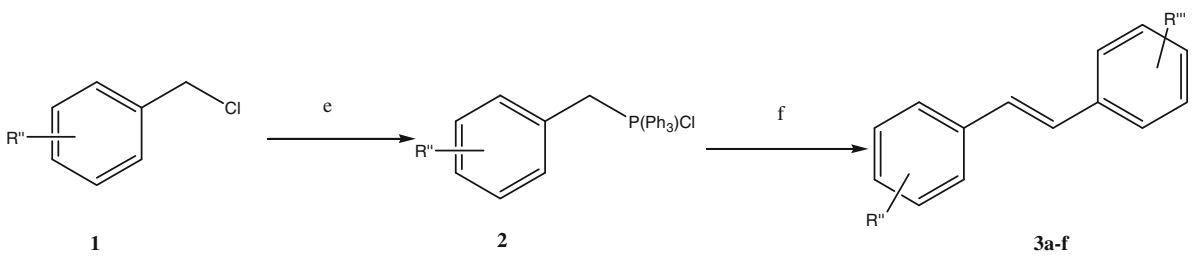

Table 1 Trans stilbenes 3a-f, and standard melphalan<smiles></smiles>

\begin{tabular}{|c|c|c|c|c|c|c|}
\hline \multirow[t]{2}{*}{ S. no } & \multirow[t]{2}{*}{$\mathrm{R}^{\prime \prime}$} & \multirow[t]{2}{*}{$\mathrm{R}^{\prime \prime \prime}$} & \multirow[t]{2}{*}{$\mathrm{MP}\left({ }^{\circ} \mathrm{C}\right)$} & \multicolumn{3}{|l|}{$\mathrm{IC}_{50}(\mu \mathrm{g} / \mathrm{ml})$} \\
\hline & & & & L1210 & Molt4/C8 & CEM \\
\hline $3 \mathbf{a}$ & $4-\mathrm{F}$ & $4-\mathrm{OCH}_{3}$ & $136-138$ & $26 \pm 10$ & $>200$ & $156 \pm 75$ \\
\hline $3 \mathbf{b}$ & $4-\mathrm{CH}_{3}$ & $4-\mathrm{N}\left(\mathrm{CH}_{3}\right)_{2}$ & $139-140$ & $143 \pm 4.0$ & $>200$ & $>200$ \\
\hline $3 c$ & $4-\mathrm{CH}_{3}$ & $4-\mathrm{OCH}_{3}$ & $143-145$ & $>200$ & $>200$ & $>200$ \\
\hline 3d & $4-\mathrm{F}$ & $2-\mathrm{F}$ & $115-121$ & $62 \pm 15$ & $61 \pm 9.0$ & $83 \pm 11$ \\
\hline $3 e$ & $4-\mathrm{CH}_{3}$ & $2-\mathrm{F}$ & $135-138$ & $72 \pm 1.0$ & $30 \pm 7.0$ & $78 \pm 18$ \\
\hline $3 f$ & $4-\mathrm{F}$ & $\mathrm{H}$ & $121-123$ & $>200$ & $>200$ & $>200$ \\
\hline Melphalan & - & - & - & $2.1 \pm 0.02$ & $3.2 \pm 0.6$ & $2.5 \pm 0.2$ \\
\hline
\end{tabular}

$\mathrm{IC}_{50}: 50 \%$ inhibitory concentration 
Scheme 2 General procedure for the preparation of $\mathbf{6 a - c}$, $\mathbf{8 a}-\mathbf{g}, 9 \mathbf{a}$ and $\mathbf{b}$ reagents and conditions: $a$ triethyl amine, acetic anhydride, dil $\mathrm{HCl}$ $b \mathrm{H}_{2} \mathrm{SO}_{4}, \mathrm{MeOH} c \mathrm{SOCl}_{2}$, benzene, $d$ Amines, $2 \mathrm{~h}$, rt<smiles>CC(=O)OC(=Cc1ccccc1)c1ccccc1</smiles>

Biological evaluation

The effects of 18 stilbene analogues and 4 analogues of Schiff derivatives as bioisosteres of stilbene on cell growth are summarized in Tables 1, 2 and 3. This group of compounds include 6 trans stilbenes (3a-f) (Table 1), 12 cis stilbene derivatives with substitution on the bridge connecting two phenyl rings (6a-c, 8a-g, 9a and b) (Table 2), and bioisosteres of stilbenes as Schiff derivatives (12a-f) (Table 3).

All the trans stilbenes $(\mathbf{3 a}-\mathbf{f})$ were poorly cytostatic (Table 1). The $\mathrm{IC}_{50}$ values ranged between 26 and $>200 \mu \mathrm{M}$.

Regarding the cis stilbenes with substitutions on the olefinic bridge (Table 2), there was not much improvement in cytostatic activity. In separate experiments, a $\mathrm{COOH}$ group was introduced on the olefinic carbon linkage, and this resulted in the formation of compounds $\mathbf{6 a}, \mathbf{b}$ and $\mathbf{c}\left(\mathrm{IC}_{50} 37\right.$ to $\left.>200 \mu \mathrm{g} / \mathrm{ml}\right)$. However, when the $-\mathrm{COOH}$ group of the compound was converted to a methyl ester (compound 9a and b) or the $N$-diethylamide (8c), the cytostatic activity somewhat increased, as particularly noted for $\mathbf{8 c}$ and 9a. However, the $N$-methylamide derivatives $(\mathbf{8} \mathbf{a}, \mathbf{b}, \mathbf{f}$ and $\mathbf{g})$ were clearly less cytostatic.

In the third set of modifications, we designed Schiff derivatives as a bioisosteric replacement of trans stilbenes. All these compounds (12a-d) turned out to be much more potent than trans stilbenes and cis restricted stilbenes in inhibiting tumor cell proliferation $\left(\mathrm{IC}_{50}: 0.77-10 \mu \mathrm{g} / \mathrm{ml}\right)$. Several cytostatic activities were as potent as those found for melphalan. These data justify more in-depth studies on this type of compounds by introducing a variety of modifications on the aryl moieties of these molecules.

\section{Conclusion}

Several trans and cis stilbenes with substitution on the olefinic bridge were synthesized in an effort to obtain substances that could be more readily formulated. None of these compounds emerged as cytostatic compounds. But 
Table 2 Compounds $6 \mathbf{a}-\mathbf{c}, 8 \mathbf{8}-\mathbf{g}$ and $9 \mathbf{a}$ and $\mathbf{b}$<smiles>[Y]/C(=C/c1ccccc1)c1ccccc1</smiles>

\begin{tabular}{|c|c|c|c|c|c|c|}
\hline \multirow[t]{2}{*}{ S. no } & \multirow[t]{2}{*}{$\mathrm{Y}$} & \multirow[t]{2}{*}{$\mathrm{R}^{\prime}$} & \multirow[t]{2}{*}{$\mathrm{MP}\left({ }^{\circ} \mathrm{C}\right)$} & \multicolumn{3}{|c|}{$\mathrm{IC}_{50}(\mu \mathrm{g} / \mathrm{ml})$} \\
\hline & & & & L1210 & Molt4/C8 & CEM \\
\hline $6 \mathbf{a}$ & $\mathrm{COOH}$ & $2-\mathrm{Cl}$ & $105-108$ & $76 \pm 12$ & $37 \pm 24$ & $93 \pm 10$ \\
\hline $6 b$ & $\mathrm{COOH}$ & $2-\mathrm{F}$ & $170-172$ & $>200$ & $89 \pm 2.0$ & $>200$ \\
\hline $6 c$ & $\mathrm{COOH}$ & $2-\mathrm{OH}$ & $139-140$ & $>200$ & $>200$ & $>200$ \\
\hline $8 \mathbf{a}$ & $\mathrm{CONHCH}_{3}$ & $2-\mathrm{Cl}$ & $100-103$ & $65 \pm 25$ & $77 \pm 0.0$ & $60 \pm 1$ \\
\hline $8 b$ & $\mathrm{CONHCH}_{3}$ & $4-\mathrm{OCH}_{3}$ & $182-185$ & $68 \pm 47$ & $166 \pm 16$ & $128 \pm 58$ \\
\hline $8 c$ & $\mathrm{CON}\left(\mathrm{C}_{2} \mathrm{H}_{5}\right)_{2}$ & $2-\mathrm{Cl}$ & $60-63$ & $24 \pm 11$ & $14 \pm 2$ & $18 \pm 12$ \\
\hline $8 d$ & $\mathrm{CONHC}_{2} \mathrm{H}_{5}$ & $\mathrm{H}$ & $99-102$ & $132 \pm 40$ & $112 \pm 44$ & $26 \pm 8$ \\
\hline $8 e$ & $\mathrm{CONHC}_{2} \mathrm{H}_{5}$ & $4-\mathrm{OCH}_{3}$ & $135-138$ & $148 \pm 45$ & $150 \pm 71$ & $116 \pm 87$ \\
\hline $8 f$ & $\mathrm{CONHCH}_{3}$ & $2-\mathrm{F}$ & $77-80$ & $85 \pm 9$ & $72 \pm 3$ & $89 \pm 15$ \\
\hline $8 g$ & $\mathrm{CONHCH}_{3}$ & $2-\mathrm{OH}$ & $69-70$ & $>200$ & $>200$ & $>200$ \\
\hline $9 a$ & $\mathrm{COOCH}_{3}$ & $2-\mathrm{Cl}$ & $113-115$ & $25 \pm 10$ & $16 \pm 1$ & $19 \pm 7$ \\
\hline $9 b$ & $\mathrm{COOCH}_{3}$ & $4-\mathrm{OCH}_{3}$ & $127-130$ & $66 \pm 46$ & $71 \pm 9$ & $71 \pm 46$ \\
\hline
\end{tabular}

$\mathrm{IC}_{50}: 50 \%$ inhibitory concentration

Scheme 3 General procedure for the preparation of 12a-d reagents and conditions: $g$ toluene, Dean Stark apparatus

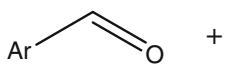

$\mathrm{H}_{2} \mathrm{~N} \nearrow_{A r^{\prime}}$

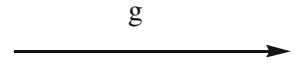

11<smiles>[Al]C=N[Al]</smiles>

12a-d

Table 3 Compounds 12a-d<smiles>[Al]C=N[Al]</smiles>

\begin{tabular}{|c|c|c|c|c|c|c|}
\hline \multirow[t]{2}{*}{ S. no } & \multirow[t]{2}{*}{$\mathrm{Ar}$} & \multirow[t]{2}{*}{$\mathrm{Ar}^{\prime}$} & \multirow[t]{2}{*}{$\operatorname{MP}\left({ }^{\circ} \mathrm{C}\right)$} & \multicolumn{3}{|c|}{$\mathrm{IC}_{50}(\mu \mathrm{g} / \mathrm{ml})$} \\
\hline & & & & L1210 & Molt4/C8 & CEM \\
\hline $12 \mathrm{a}$ & 2-Cl-phenyl & 3-Cl-4-F-phenyl & $57-55$ & $6.4 \pm 2.9$ & $3.9 \pm 3.0$ & $10 \pm 4$ \\
\hline $12 b$ & 2-Cl-phenyl & 4-F-phenyl & $35-37$ & $6.0 \pm 0.7$ & $0.77 \pm 0.22$ & $2.1 \pm 1.7$ \\
\hline $12 \mathrm{c}$ & 2-Cl-phenyl & 4-Br-phenyl & $38-40$ & $2.1 \pm 1.4$ & $1.5 \pm 0.9$ & $4.5 \pm 2.6$ \\
\hline 12d & 2-thiophene & 3-Cl-4-F-phenyl & $40-42$ & $3.2 \pm 1.8$ & $16 \pm 8$ & $25 \pm 18$ \\
\hline
\end{tabular}

$\mathrm{IC}_{50}: 50 \%$ inhibitory concentration 
Schiff derivatives emerged as interesting antiproliferative compounds against the three tumor cell lines evaluated.

\section{Experimental}

\section{Chemistry}

The solvents (AR grades) were obtained from Sd Fine Chem., Mumbai, and E. Merck, Mumbai. The reagents (puriss grade) were obtained from Fluka and E. Merck. FT-IR spectra were recorded in $\mathrm{KBr}$ powder on a Jasco V410 FT-IR spectrometer by diffuse reflectance technique. ${ }^{1} \mathrm{H} /{ }^{13} \mathrm{C}$ NMR spectra were measured in $\mathrm{CDCl}_{3}$ and DMSOd6 on a Bruker Ultraspec AMX $400 \mathrm{MHz}$ spectrometer. The reported chemical shifts were against that of TMS. Elemental Analysis was carried out at CDRI, Lucknow on Elementar Vario EL III, Carlo Erba 1108.

\section{General procedure for the preparation of stilbenes $\mathbf{3 a}-\boldsymbol{f}$}

A stirred solution of benzyl chloride $\mathbf{1}(2.88 \mathrm{~g}, 31.7 \mathrm{mmol})$ in acetonitrile $(20 \mathrm{ml})$ was treated with triphenylphosphine $(8.57 \mathrm{~g}, 32.7 \mathrm{mmol})$, and the mixture was refluxed with stirring for $12 \mathrm{~h}$ and then evaporated. The crude product was purified by crystallization from chloroform and ether, affording $95 \%$ yield as a white solid $\mathbf{2}$. Sodium hydride (72 $\mathrm{mg}, 3 \mathrm{mmol}$ ) was added in portions to a well-stirred suspension of phosphonium chloride $2(2 \mathrm{mmol})$ and aryl aldehyde $(2 \mathrm{mmol})$ in benzene $(20 \mathrm{ml})$ at $0-5^{\circ} \mathrm{C}$, and the mixture was allowed to warm up to room temperature. After an additional stirring for $16 \mathrm{~h}$, excess sodium hydride was quenched by the addition of methanol $(1 \mathrm{ml})$, after which $30 \mathrm{ml}$ of chloroform and water was added. The organic and aqueous layers were then separated. Distilled of the organic layer, the residue was purified by preparative TLC using 5\% ethanol in hexane as the eluent.

\section{(E)-1-(4-Fluorophenyl)-2-(4-methoxyphenyl)-ethene (3a)}

Yield 25\%; FT-IR $v_{\max } \mathrm{cm}^{-1}$ (KBr): 3042-3017, 2924-2844, 1604, 1573, 1511 and 1463. Anal. Calcd for $\mathrm{C}_{15} \mathrm{H}_{13} \mathrm{OF}$ : C, 78.93; H, 5.74. Found: C, 78.25; H, 5.55.

\section{(E)-1-(4-Methylphenyl)-2-(4-dimethylaminophenyl)-}

ethene $(3 \boldsymbol{b})$

Yield 23\%; Anal. Calcd for $\mathrm{C}_{17} \mathrm{H}_{19} \mathrm{~N}$ : C, 86.03; H, 8.07; N, 5.90 Found: C, 86.25; H, 7.89; N, 6.05. FT-IR $v_{\max } \mathrm{cm}^{-1}$ (KBr): 3197-3021，2916-2808，1600，1528，1437 and 1363. ${ }^{1} \mathrm{H}-\mathrm{NMR}\left(\mathrm{CDCl}_{3}\right) \delta ; 7.42(4 \mathrm{H}, \mathrm{m}), 7.13(2 \mathrm{H}, \mathrm{d}$,
$J=8 \mathrm{~Hz}), 6.99(1 \mathrm{H}, \mathrm{d}), 6.92(1 \mathrm{H}, \mathrm{d}), 6.71(2 \mathrm{H}, \mathrm{d}$, $J=8.8 \mathrm{~Hz}), 2.98\left(6 \mathrm{H}, \mathrm{s},-\mathrm{N}\left(\mathrm{CH}_{3}\right)_{2}\right), 2.35\left(3 \mathrm{H}, \mathrm{s}, \mathrm{CH}_{3}\right)$.

\section{(E)-1-(4-Methylphenyl)-2-(4-methoxyphenyl)-ethene (3c)}

Yield 25\%; Anal. Calcd for $\mathrm{C}_{16} \mathrm{H}_{16} \mathrm{O}$ : C, 85.68; H, 7.19. Found: C, 85.34; H, 6.99. FT-IR $v_{\max } \mathrm{cm}^{-1}(\mathrm{KBr})$ : 3049-3021, 2954-2916, 1603, 1515, 1439 and 1377. ${ }^{1} \mathrm{H}-$ $\operatorname{NMR}\left(\mathrm{CDCl}_{3}\right) \delta ; 7.44(2 \mathrm{H}, \mathrm{d}, J=8.8 \mathrm{~Hz}), 7.38(2 \mathrm{H}, \mathrm{d}$, $J=8 \mathrm{~Hz}), \quad 7.15 \quad(2 \mathrm{H}, \quad \mathrm{d}, \quad J=8 \mathrm{~Hz}), 7.01 \quad(1 \mathrm{H}, \quad \mathrm{d}$, $J=16.4 \mathrm{~Hz}), 6.93(1 \mathrm{H}, \mathrm{d}, J=16.4 \mathrm{~Hz}), 6.89(2 \mathrm{H}, \mathrm{d}, J=$ $8.8 \mathrm{~Hz}), 3.83\left(3 \mathrm{H}, \mathrm{s}, \mathrm{OCH}_{3}\right), 2.36\left(3 \mathrm{H}, \mathrm{s},-\mathrm{CH}_{3}\right)$.

\section{(E)-1-(4-Fluorophenyl)-2-(2-fluorophenyl)-ethene (3d)}

Yield 26\%; Anal. Calcd for $\mathrm{C}_{14} \mathrm{H}_{10} \mathrm{~F}_{2}$ : C, 77.77; H, 4.66. Found: C, 77.34; H, 4.29. FT-IR $v_{\max } \mathrm{cm}^{-1}(\mathrm{KBr})$ : 3077-3055, 2918, 1590, 1484 and 1438.

\section{(E)-1-(4-Methylphenyl)-2-(2-fluorophenyl)-ethene (3e)}

Yield 24\%; Anal. Calcd for $\mathrm{C}_{15} \mathrm{H}_{13} \mathrm{~F}$ : C, 84.88; H, 6.17. Found: C, 84.64; H, 5.99. FT-IR $v_{\max } \mathrm{cm}^{-1}(\mathrm{KBr})$ : 3081-3056, 2953-2919, 1590, 1483 and 1437.

\section{(E)-1-(4-Fluorophenyl)-2-phenyl-ethene (3f)}

Yield 22\%; Anal. Calcd for $\mathrm{C}_{14} \mathrm{H}_{14} \mathrm{~F}$ : C, 84.82; H, 5.59. Found: C, 84.67; H, 5.25. FT-IR $v_{\max } \mathrm{cm}^{-1}(\mathrm{KBr})$ : 3080-3023, 2953-2849, 1593, 1508 and 1448.

\section{General procedure for the preparation}

of compounds $(\boldsymbol{6} \boldsymbol{a}-\boldsymbol{c})$

A mixture of phenyl acetic acid $4(2 \mathrm{mmol})$ substituted benzaldehyde $5(2 \mathrm{mmol})$ and triethylamine $(0.5 \mathrm{ml})$ in acetic anhydride $(5 \mathrm{ml})$ was heated under reflux for $12 \mathrm{~h}$ and poured into hot saturated sodium carbonate solution $(50 \mathrm{ml})$ and left overnight. The mixture was extracted with ether $(2 \times 50 \mathrm{ml})$, the ether extracts were discarded, and the aqueous solution was acidified with dilute $\mathrm{HCl}$, after which the precipitated product was filtered and dried. Recrystallization from ethyl acetate-hexane gave the pure products.

\section{(E)-3-(2-Chlorophenyl)-2-phenyl-prop-2-enoic acid (6a)}

Yield 33\%; Anal. Calcd for $\mathrm{C}_{15} \mathrm{H}_{11} \mathrm{O}_{2} \mathrm{Cl}$ : C, 69.64; H, 4.29. Found: $\mathrm{C}, 69.25 ; \mathrm{H}, 4.15$. FT-IR $v_{\max } \mathrm{cm}^{-1}(\mathrm{KBr})$ : 3250-2500, 1685, 1425 and 1266. ${ }^{1} \mathrm{H}-\mathrm{NMR}\left(\mathrm{CDCl}_{3}\right) \delta$; $8.20(1 \mathrm{H}, \mathrm{s}), 7.37(1 \mathrm{H}, \mathrm{d}, J=8 \mathrm{~Hz}), 7.31-7.26(3 \mathrm{H}, \mathrm{m})$, $7.21-7.19(2 \mathrm{H}, \mathrm{m}), 7.15(\mathrm{t}, J=15.2 \mathrm{~Hz}), 6.90(1 \mathrm{H}, \mathrm{t}$, 
$J=15.2 \mathrm{~Hz}), 6.76(1 \mathrm{H}, \mathrm{d}, J=8 \mathrm{~Hz}) . \mathrm{MS}: m / z(\%) 259$

$(\mathrm{M}+1)$.

(E)-3-(2-Fluorophenyl)-2-phenyl-prop-2-enoic acid (6b)

Yield 44\%; Anal. Calcd for $\mathrm{C}_{15} \mathrm{H}_{11} \mathrm{O}_{2} \mathrm{~F}$ : C, 74.37; H, 4.58. Found: C, 74.09; H, 4.41. FT-IR $v_{\max } \mathrm{cm}^{-1}(\mathrm{KBr})$ : 3200-2600, 1682 and $1620 .{ }^{1} \mathrm{H}-\mathrm{NMR}(\mathrm{CDCl} 3) \delta ; 8.15(1 \mathrm{H}$, s), 7.38-7.30 (3H, m), 7.25-7.21 (3H, m), 7.06-7.01 (1H, m), 6.81-6.74 (2H, m). MS: $\mathrm{m} / z(\%) 243(\mathrm{M}+2)$.

(E)-3-(2-Hydroxyphenyl)-2-phenyl-prop-2-enoic acid (6c)

Yield 46\%; Anal. Calcd for $\mathrm{C}_{15} \mathrm{H}_{12} \mathrm{O}_{3}$ : C, 74.99; H, 5.03. Found: C, 75.12; H, 4.79. FT-IR $v_{\max } \mathrm{cm}^{-1}(\mathrm{KBr}): 3405$, 3109-3051, 2970, 1713, 1613, 1454 and 1262.

\section{General procedure for the preparation}

of compounds $(8 \boldsymbol{a}-\mathbf{g})$

A mixture of carboxylic acid $6(0.5 \mathrm{mmol})$ and thionyl chloride $(1 \mathrm{ml})$ and benzene $(10 \mathrm{ml})$ was refluxed for $6 \mathrm{~h}$. The excess thionyl chloride and benzene were removed at reduced pressure, and the residue 7 was subsequently mixed with amines $(5 \mathrm{ml})$ and kept at room temperature for $2 \mathrm{~h}$. The precipitated product was filtered, washed subsequently with $2 \% \mathrm{NaOH}$ solution and water and dried. The product was purified with suitable solvent.

(E)-N-Methyl-3-(2-chlorophenyl)-2-(phenyl)prop-2-enamide $(8 \boldsymbol{a})$

Yield 46\%; Anal. Calcd for $\mathrm{C}_{16} \mathrm{H}_{14} \mathrm{ONCl}$ : C, 70.72; $\mathrm{H}$, 5.19; N, 5.15. Found: C, 70.15; H, 5.07; N, 5.21. FT-IR $v_{\max } \mathrm{cm}^{-1}(\mathrm{KBr}): 3320,3078-3023,2940-2931,1654$, 1617, 1403 and 1269. ${ }^{1} \mathrm{H}-\mathrm{NMR}\left(\mathrm{CDCl}_{3}\right) \delta ; 8.01(1 \mathrm{H}, \mathrm{s})$, 7.35-7.33 (3H, m), 7.26 (2H, s), 7.20-7.18 (2H, m), $7.10-7.06(1 \mathrm{H}, \mathrm{m}), 6.85(1 \mathrm{H}, \mathrm{t}, J=18.8 \mathrm{~Hz}), 6.65(1 \mathrm{H}, \mathrm{d}$, $J=7.6 \mathrm{~Hz}), 5.55\left(1 \mathrm{H}, \mathrm{s}, \mathrm{br}, \mathrm{NH}, \mathrm{D}_{2} \mathrm{O}\right.$ exchangeable $), 2.88$ $\left(3 \mathrm{H}, \mathrm{s},-\mathrm{CH}_{3}\right)$. MS: $m / z(\%) 272(\mathrm{M}+1)$.

(E)-N-Methyl-3-(4-methoxyphenyl)-2-(phenyl)prop-2-enamide $(\boldsymbol{8 b})$

Yield 48\%; Anal. Calcd for $\mathrm{C}_{17} \mathrm{H}_{17} \mathrm{NO}_{2}$ : C, 76.38; $\mathrm{H}, 6.41$; $\mathrm{N}$, 5.24. Found: C, 76.19; H, 6.25; N, 5.29. FT-IR $v_{\max }$ $\mathrm{cm}^{-1}$ (KBr): 3442, 3099-3006, 2958-2836, 1661, 1599, 1514, 1460 and $1405 .{ }^{1} \mathrm{H}-\mathrm{NMR}\left(\mathrm{CDCl}_{3}\right) \delta ; 7.82(1 \mathrm{H}, \mathrm{s})$, 7.48-7.42 (3H, m), 7.26-7.24 (2H, m), $6.90(2 \mathrm{H}, \mathrm{d}$, $J=8.8 \mathrm{~Hz}), 6.64(2 \mathrm{H}, \mathrm{d}, J=8.8 \mathrm{~Hz}), 5.44(1 \mathrm{H}, \mathrm{s}, \mathrm{br}, \mathrm{NH}$, $\mathrm{D}_{2} \mathrm{O}$ exchangeable), $3.74\left(3 \mathrm{H}, \mathrm{s}, \mathrm{OCH}_{3}\right), 2.84\left(3 \mathrm{H}, \mathrm{s}, \mathrm{CH}_{3}\right)$.
(E)-N-Diethyl-3-(2-chlorophenyl)-2-(phenyl)prop-2-enamide $(8 \mathrm{c})$

Yield 23\%; Anal. Calcd for $\mathrm{C}_{19} \mathrm{H}_{20} \mathrm{NOCl}$ : C, 72.72; H, 6.42; N, 4.46. Found: C, 72.02; H, 6.99; N, 4.51. FT-IR $v_{\max } \mathrm{cm}^{-1}(\mathrm{KBr}): 3442,3077-3020,2986-2873,1621$, 1573,1470 and $1433 .{ }^{1} \mathrm{H}-\mathrm{NMR}\left(\mathrm{CDCl}_{3}\right) \delta ; 7.39(1 \mathrm{H}, \mathrm{d}$, $J=8 \mathrm{~Hz}), \quad 7.25-7.21 \quad(5 \mathrm{H}, \mathrm{m}), \quad 7.16-7.11 \quad(1 \mathrm{H}, \mathrm{m})$, 6.98-6.95 (1H, m), $6.79(1 \mathrm{H}, \mathrm{s}), 3.48(2 \mathrm{H}, \mathrm{d}, J=8 \mathrm{~Hz})$, $3.36(2 \mathrm{H}, \mathrm{d}, J=8 \mathrm{~Hz}), 1.18(3 \mathrm{H}, \mathrm{t}, J=12 \mathrm{~Hz}), 1.02(3 \mathrm{H}$, $\mathrm{t}, J=12 \mathrm{~Hz})$.

\section{(E)-N-Ethyl-3-(phenyl)-2-(phenyl)-prop-2-enamide (8d)}

Yield 44\%; Anal. Calcd for $\mathrm{C}_{17} \mathrm{H}_{16} \mathrm{NOCl}$ : C, 71.45; H, 5.64; N, 4.90. Found: C, 69.98; H, 5.25; N, 5.01. FT-IR $v_{\max } \mathrm{cm}^{-1}(\mathrm{KBr}): 3329,3077-3018,2974-2871,1654$, 1528, 1493 and 1261.

(E)-N-Ethyl-3-(4-methoxyphenyl)-2-(phenyl)prop-2-enamide (8e)

Yield 44\%; Anal. Calcd for $\mathrm{C}_{18} \mathrm{H}_{19} \mathrm{NO}_{2}$ : C, 76.84; $\mathrm{H}, 6.81$; N, 4.98. Found: C, 76.02; H, 6.53; N, 5.01. FT-IR $v_{\max }$ $\mathrm{cm}^{-1}$ (KBr): 3303, 3053-3004, 2966-2842, 1650, 1606, 1525,1459 and 1349. ${ }^{1} \mathrm{H}-\mathrm{NMR}\left(\mathrm{CDCl}_{3}\right) \delta ; 7.81(1 \mathrm{H}, \mathrm{s})$, 7.49-7.43 (3H, m), 7.27-7.25 (2H, m), $6.90(2 \mathrm{H}, \mathrm{d}$, $J=8.8 \mathrm{~Hz}), 6.65(2 \mathrm{H}, \mathrm{d}, J=8.8 \mathrm{~Hz}), 5.41(1 \mathrm{H}, \mathrm{s}, \mathrm{br}, \mathrm{NH}$, $\mathrm{D}_{2} \mathrm{O}$ exchangeable), $3.74\left(3 \mathrm{H}, \mathrm{s}, \mathrm{OCH}_{3}\right), 3.37-3.30(2 \mathrm{H}, \mathrm{q}$, $\left.\mathrm{CH}_{2}\right), 1.07\left(3 \mathrm{H}, \mathrm{t}, J=14.5 \mathrm{~Hz}, \mathrm{CH}_{3}\right)$.

(E)-N-Methyl-3-(2-fluorophenyl)-2-(phenyl)prop-2-enamide (8f)

Yield 45\%; Anal. Calcd for $\mathrm{C}_{16} \mathrm{H}_{14} \mathrm{NOF}$ : C, 75.28; H, 5.53; $\mathrm{N}$, 5.49. Found: C, 69.89; H, 5.36; N, 5.35. FT-IR $v_{\max }$ $\mathrm{cm}^{-1}$ (KBr): 3316, 3079-3022, 2933, 1656, 1619, 1454 and 1271. ${ }^{1} \mathrm{H}-\mathrm{NMR}\left(\mathrm{CDCl}_{3}\right): \delta ; 8.01(1 \mathrm{H}, \mathrm{s}), 7.43-7.39$ (3H, m), 7.24-7.22 (2H, m), 7.17-7.15 (1H, m), 7.01-6.97 $(1 \mathrm{H}, \quad \mathrm{m}), 6.74(1 \mathrm{H}, \quad \mathrm{t}, \quad J=15.2 \mathrm{~Hz}), 6.61(1 \mathrm{H}, \mathrm{t}$, $J=15.2 \mathrm{~Hz}), 5.53\left(1 \mathrm{H}, \mathrm{s}, \mathrm{br}, \mathrm{NH}, \mathrm{D}_{2} \mathrm{O}\right.$ exchangeable $)$, $2.86\left(3 \mathrm{H}, \mathrm{s},-\mathrm{CH}_{3}\right)$.

(E)-N-Methyl-3-(2-hydroxyphenyl)-2-(phenyl)prop-2-enamide (8g)

Yield 38\%; Anal. Calcd for $\mathrm{C}_{16} \mathrm{H}_{15} \mathrm{NO}_{2}$ : C, 75.87; H, 5.97; $\mathrm{N}, 5.53$. Found: C, 75.19; H, 5.55; N, 5.61. FT-IR $v_{\max }$ $\mathrm{cm}^{-1}$ (KBr): 3406, 3106-3057, 2966-2929, 1714, 1667, 1612, 1488 and 1454. ${ }^{1} \mathrm{H}-\mathrm{NMR}(\mathrm{CDCl} 3) \delta ; 8.01(1 \mathrm{H}, \mathrm{s})$, 7.35-7.33 (3H, m), $7.26(2 \mathrm{H}, \mathrm{s}), 7.20-7.18(2 \mathrm{H}, \mathrm{m})$, $7.10-7.06(1 \mathrm{H}, \mathrm{m}), 6.85(1 \mathrm{H}, \mathrm{t}, J=18.8 \mathrm{~Hz}), 6.65(1 \mathrm{H}, \mathrm{d}$, 
$J=7.6 \mathrm{~Hz}), 5.55\left(1 \mathrm{H}, \mathrm{s}, \mathrm{br}, \mathrm{NH}, \mathrm{D}_{2} \mathrm{O}\right.$ exchangeable), 2.88 $\left(3 \mathrm{H}, \mathrm{s},-\mathrm{CH}_{3}\right)$.

\section{General procedure for the preparation of compounds $9 \boldsymbol{a}$ and $\boldsymbol{b}$}

Concentrated $\mathrm{H}_{2} \mathrm{SO}_{4}(0.5 \mathrm{ml})$ was added to a stirred solution of carboxylic acid $(0.5 \mathrm{mmol})$ in absolute methanol $(20 \mathrm{ml})$ and the mixture was heated under reflux for $6 \mathrm{~h}$. Excess of methanol was removed by evaporation, and the residue was poured into ice water. The product was extracted with ether $(2 \times 20 \mathrm{ml})$, and the combined extracts were washed with $2 \%$ aqueous $\mathrm{NaOH}$ solution $(2 \times 50 \mathrm{ml})$ followed by water $(200 \mathrm{ml})$. Evaporation of ether layer gave the desired product.

(E)-Methyl-3-(2-chlorophenyl)-2-(phenyl)-prop-

2-enoate (9a)

Yield 48\%; Anal. Calcd for $\mathrm{C}_{16} \mathrm{H}_{13} \mathrm{O}_{2} \mathrm{Cl}$ : C, 70.46; H, 4.80 . Found: C, 69.99; H, 4.98. FT-IR $v_{\max } \mathrm{cm}^{-1}(\mathrm{KBr}): 3057$, 2991-2848, 1704, 1570, 1496 and 1387.

(E)-Methyl-3-(4-methoxyphenyl)-2-(phenyl)-prop2-enoate $(9 b)$

Yield 45\%; Anal. Calcd for $\mathrm{C}_{17} \mathrm{H}_{16} \mathrm{O}_{3}$ : C, 76.10; H, 6.10. Found: C, 75.98; H, 5.79. FT-IR $v_{\max } \mathrm{cm}^{-1}(\mathrm{KBr}): 3055$, 2954-2837, 1704, 1597, 1513 and 1385.

\section{General procedure for the preparation of Schiff} derivatives $(12 a-d)$

A solution of aldehyde $10(1 \mathrm{mmol})$ and aniline $\mathbf{1 1}$ $(1 \mathrm{mmol})$ in toluene $(5 \mathrm{ml})$ were heated to reflux in a Dean Stark apparatus for $16 \mathrm{~h}$. After the solvent was removed in vacuo, the crude product was recrystallised from ethanol to give $45-50 \%$ yield.

\section{(E)-3-Chloro-N-(2-chlorobenzyledene)-4-fluoro benzenamine (12a)}

Yield 45\%; Anal. Calcd for $\mathrm{C}_{13} \mathrm{H}_{8} \mathrm{Cl}_{2} \mathrm{~N}$ : C, 58.24; $\mathrm{H}, 3.01$; $\mathrm{N}$, 5.22. Found: C, 58.01; H, 2.99; N, 5.31. IR $(\mathrm{KBr}) \mathrm{cm}^{-1}$ : 3089-2996, 2922, 1620 and 1494. ${ }^{1} \mathrm{H}-\mathrm{NMR}\left(\mathrm{CDCl}_{3}\right)$ : $\delta=8.89(1 \mathrm{H}, \mathrm{s}), 8.20(1 \mathrm{H}, \mathrm{d}, J=8 \mathrm{~Hz}), 7.46-7.43(2 \mathrm{H}$, $\mathrm{m}), 7.41-7.36(1 \mathrm{H}, \mathrm{m}), 7.33-7.31(1 \mathrm{H}, \mathrm{m}), 7.21-7.12(2 \mathrm{H}$, m). MS: $m / z(\%): 268(\mathbf{M})$.

\section{(E)-N-(2-Chlorobenzyledene)-4-fluorobenzenamine (12b)}

Yield 51\%; Anal. Calcd for $\mathrm{C}_{13} \mathrm{H}_{9} \mathrm{ClN}$ : C, 66.82; $\mathrm{H}, 3.88$; $\mathrm{N}, 5.99$. Found: C, 66.79; H, 3.58; N, 6.31. FT-IR $v_{\max }$
$\mathrm{cm}^{-1}(\mathrm{KBr}):$ 3075-2997, 2918-2848, 1617 and 1498. ${ }^{1} \mathrm{H}-$ $\operatorname{NMR}\left(\mathrm{CDCl}_{3}\right): \delta=8.9(1 \mathrm{H}, \mathrm{s}), 8.22(1 \mathrm{H}, \mathrm{d}, J=7.6 \mathrm{~Hz})$, 7.47-7.35 (3H, m), 7.27-7.23 (2H, m), 7.13-7.08 (2H, m), $6.85(1 \mathrm{H}, \mathrm{t}, J=18.8 \mathrm{~Hz}), 6.65(1 \mathrm{H}, \mathrm{d}, J=7.6 \mathrm{~Hz}), 5.55$ $(1 \mathrm{H}, \mathrm{s}, \mathrm{br}, \mathrm{NH}), 2.88\left(3 \mathrm{H}, \mathrm{s},-\mathrm{CH}_{3}\right)$.

\section{(E)-N-(2-Chlorobenzyledene)-4-bromobenzenamine (12c)}

Yield 52\%; Anal. Calcd for $\mathrm{C}_{13} \mathrm{H}_{9} \mathrm{ClNBr}$ : C, 53.01; $\mathrm{H}$, 3.08; N, 4.75. Found: C, 52.75; H, 2.94; N, 4.94. FT-IR $v_{\max } \mathrm{cm}^{-1}(\mathrm{KBr}): 3061-3018,2918-2848,1618$ and 1484. ${ }^{1} \mathrm{H}-\mathrm{NMR}(\mathrm{CDCl} 3): \delta=8.92-8.90(1 \mathrm{H}, \mathrm{m}), 7.26-7.22(1 \mathrm{H}$, $\mathrm{m}), 7.56-7,52(2 \mathrm{H}, \mathrm{m}), 7.47-7.38(2 \mathrm{H}, \mathrm{m}), 7.26(1 \mathrm{H}, \mathrm{d}$, $J=9.6 \mathrm{~Hz}), 7.16-7.12(2 \mathrm{H}, \mathrm{m})$.

(E)-3-Chloro-4-fluoro-N-(thiophen-2-ylmethylene)benzenamine (12d)

Yield 56\%; Anal. Calcd for $\mathrm{C}_{11} \mathrm{H}_{7} \mathrm{ClFNS}$ : C, 55.12; $\mathrm{H}$, 2.94; N, 5.84. Found: C, 55.01; H, 3.01; N, 5.55. FT-IR $v_{\max } \mathrm{cm}^{-1}(\mathrm{KBr}): 3060,2954,2886,1615$ and $1492 .{ }^{1} \mathrm{H}-$ NMR $(\mathrm{CDCl} 3): \delta=8.53 \quad(1 \mathrm{H}, \quad \mathrm{s}), \quad 7.54 \quad(1 \mathrm{H}, \quad \mathrm{d}$, $J=5.2 \mathrm{~Hz}), 7.50(1 \mathrm{H}, \mathrm{d}, J=3.6 \mathrm{~Hz}), 7.29-7.26(1 \mathrm{H}, \mathrm{m})$, 7.17-7.08 (3H, m).

\section{Cytostatic activity}

The methodology for measuring the cytostatic activity in Molt 4/C8, CEM and L1210 assays has been published previously (Baraldi et al., 2004). In brief, varying concentrations of compounds were incubated at $37^{\circ} \mathrm{C}$ with the cells for 72 (human Molt 4/C8 or CEM T-lymphocytes) or $48 \mathrm{~h}$ (murine L1210 cells). After the incubation period, the cell number was counted by a coulter counter (Harpenden Herz, UK).

\section{References}

Baraldi PG, Del Carmen Nunez M, Tabrizi MA, De Clercq E, Balzarini J, Bermejo J, Estévez F, Romagnoli R (2004) Design, synthesis, and biological evaluation of hybrid molecules containing $\alpha$-methylene- $\gamma$-butyrolactones and polypyrrole minor groove binders. J Med Chem 47:2877-2886

Chang TK, Lee WB, Ko HH (2000) Trans-resveratrol modulates the catalytic activity and mRNA expression of the procarcinogenactivating human cytochrome P450 1B1. Can J Physiol Pharmacol 78:874-881

Chun YJ, Kim MY, Guengerich FP (1999) Resveratrol is a selective human cytochrome P450 1A1 inhibitor. Biochem Biophys Res Commun 262:20-24

Chun YJ, Ryu SY, Jeong TC, Kim MY (2001) Mechanism based inhibition of human cytochrome P450 1A1 by rhapontigenin. Drug Metab Dispos 29:389-393

Cushman M, Nagarathnam D, Gopal D, He HM, Lin CM, Hamel E (1992) Synthesis and evaluation of analogs of (Z)-1-(4- 
methoxyphenyl)-2-(3, 4, 5-trimethoxyphenyl) ethene as potential cytotoxic and antimitotic agents. J Med Chem 35:2293-2306

Dong Z (2003) Molecular mechanism of the chemopreventive effect of resveratrol. Mutation Res 523-524:145-150

Ekena K, Katzenellenbogen JA, Katzenellenbogen BS (1998) Determinants of ligand specificity of estrogen receptor-alpha: estrogen versus androgen discrimination. J Biol Chem 273:693-699

Fontecave M (1998) Ribonucleotide reductases and radical reactions. Cell Mol Life Sci 54:684-695

Gao H, Katzenellenbogen J, Garg R, Hansch C (1999) Comparative QSAR analysis of estrogen receptor ligands. Chem Rev 99: 723-744

Heynekamp JJ, Weber WM, Hunsaker LA, Gonzales AM, Orlando RA, Deck LM, Jagt DL (2006) Substituted trans-stilbenes, including analogues of the natural product resveratrol, inhibit the human tumor necrosis factor alpha-induced activation of transcription factor nuclear factor kappaB. J Med Chem 49(24): 7182-7189

Jang M, Pezzuto JM (1999) Cancer chemopreventive activity of resveratrol. Drugs Exp Clin Res 25:65-77

Jang M, Cai L, Udeani GO, Slowing KV, Thomas CF, Beecher CW, Fong HH, Farnsworth NR, Kinghorn AD, Mehta RG, Moon RC, Pezzuto JM (1997) Cancer chemopreventive activity of resveratrol, a natural product derived from grapes. Science 275: $218-220$
Lion CJ, Matthews CS, Stevens MF, Westwell AD (2005) Synthesis, antitumor evaluation, and apoptosis-inducing activity of hydroxylated (E)-stilbenes. J Med Chem 48(4):1292-1295

Moran BW, Anderson FP, Devery A, Cloonan S, Butler WE, Varughese S, Draper SM, Kenny PT (2009) Synthesis, structural characterisation and biological evaluation of fluorinated analogues of resveratrol. Bioorg Med Chem 17(13):4510-4522

Sadler BR, Cho SJ, Ishaq KS, Chae K, Korach KS (1998) Threedimensional quantitative structure-activity relationship study of nonsteroidal estrogen receptor ligands using the comparative molecular field analysis/cross-validated $r^{2}$-guided region selection approach. J Med Chem 41:2261-2267

Sangjun S, de Jong E, Nijmeijer S, Mutarapat T, Ruchirawat S, van den Berg M, van Duursen MB (2009) Induction of cell cycle arrest in human MCF-7 breast cancer cells by cis-stilbene derivatives related to VIOXX. Toxicol Lett 186(2):115-122

Stivala LA, Savio M, Carafoli F, Perucca P, Bianchi L et al (2001) Specific structural determinants are responsible for the antioxidant activity and the cell cycle effects of resveratrol. J Biol Chem 276:22586-22594

Wright JS, Johnson ER, Dilabio GA (2001) Predicting the activity of phenolic antioxidants: theoretical method, analysis of substituent effects, application to major families of antioxidants. J Am Chem Soc 123:1173-1183 